domain protein-1/hypoxia-inducible factor-2 $\alpha$ (EPAS-1/ HIF-2 $\alpha$ ) is a catabolic transcription factor that regulates osteoarthritis $(\mathrm{OA})$ cartilage destruction. Objectives: In this study, we examined whether microRNA-365 (miR-365) affects interleukin (IL)-1 $\beta$-induced expression of catabolic factors in chondrocytes via regulation of HIF-2 $\alpha$.

Methods: Total RNA was isolated from normal and OA cartilage tissues and human chondrocytes. miR-365 expression was quantified by TaqMan assay. The effects of miR-365 on HIF-2 $\alpha$ and HIF-2 $\alpha$-modulated genes were assessed by quantitative real-time reverse polymerase chain reaction (qRT-PCR), Western blot analysis, and enzyme-linked immunosorbent assay (ELISA). Direct interaction of miR-365 with the 3 ' untranslated region (UTR) of HIF-2 $\alpha$ mRNA was examined using a luciferase reporter assay. Nitrite concentration was measured in culture medium using a Griess assay.

Results: miR-365 levels were significantly decreased in human OA cartilage relative to normal cartilage. Overexpression of miR-365 significantly suppressed IL-1 $\beta$-induced expression of HIF- $\alpha \alpha$ in human articular chondrocytes. Pharmacological inhibition of various IL-1 $1 \beta$-associated signaling pathways revealed mitogen-activated protein kinase and nuclear factor- $\mathrm{k} B$ as the primary pathways driving IL-1 $\beta$-mediated decreases in miR-365 and subsequent increase in HIF-2 $\alpha$ expression. Using a luciferase reporter assay encoding the 3'UTR of human HIF-2 $\alpha$ mRNA, we showed that overexpression of miR-365 significantly suppressed IL-1 $\beta$-induced up-regulation of HIF-2 $\alpha$. Furthermore, miR-365 overexpression significantly suppressed IL-1 $\beta$-induced expression of catabolic factors, including cyclooxygenase-2, inducible nitric oxide synthase, and matrix metalloproteinase-1, -3 and -13 , and nitric oxide (NO) production in chondrocytes.
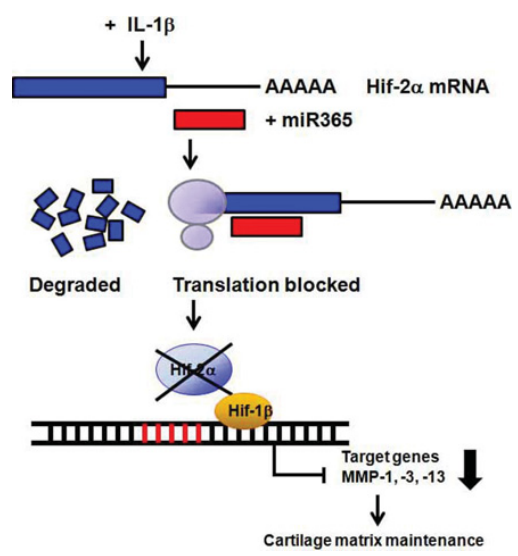

Conclusions: MiR-365 regulates IL- $1 \beta$-stimulated catabolic effects in human chondrocytes by modulating HIF-2 $\alpha$ expression.

References:

[1] Yang et al. Hypoxia-inducible factor-2alpha is a catabolic regulator of osteoarthritic cartilage destruction, Nat. Med. 16 (2010) 687-693.

[2] Akhtar et al. Haqqi, MicroRNA-27b regulates the expression of matrix metalloproteinase 13 in human osteoarthritis chondrocytes, Arthritis Rheum. 62 (2010) 1361-1371.

[3] Park et al. MicroRNA-558 regulates the expression of cyclooxygenase-2 and IL-1beta-induced catabolic effects in human articular chondrocytes, Osteoarthritis Cartilage 21 (2013) 981-989.

Acknowledgements: This study was supported by the Basic Science Research Program through the National Research Foundation (NRF) of Korea funded by the Ministry of Education (2014R1A1A2059823 and 2016R1D1A1B03932259).

Disclosure of Interest: None declared

DOI: 10.1136/annrheumdis-2017-eular.2818

\section{THU0051 ASSOCIATION BETWEEN BIOACTIVE TNF AND EULAR RESPONSE TO TNF INHIBITORS IN RHEUMATOID ARTHRITIS}

J. Poudroux ${ }^{1}$, H. Marotte ${ }^{1}$, J.-E. Gottenberg ${ }^{2}$, S. Paul ${ }^{3} .{ }^{1}$ Rheumatology, $\mathrm{CHU}$ Saint Etienne, Saint Priest en Jarez; ${ }^{2}$ Rheumatology, CHU Strasbourg, Strasbourg; ${ }^{3}$ Immunology, CHU Saint Etienne, Saint Priest en Jarez, France

Background: ROC study provided data to manage rheumatoid arthritis (RA) treatment after a failure to a first TNF blocker (1). We previously reported that high circulating TNF bioactivity was associated with good clinical response (2).

Objectives: To explore ability of TNF bioactivity to predict response to second TNF blocker.

Methods: Here, we assessed TNF bioactivity in 130 RA patients from the group rotation to a second TNF blocker at the time of randomisation and after 6 months. Clinical and biological data were recorded at baseline and 6 month with Eular response assessed at 6 month.

TNF bioactivity was assessed with HEK-Dual TNF cells adapted from our previous work (2). Due to non-normalised distribution, non parametrical analysis were performed.

Results: Baseline circulating TNF bioactivity was similar according to Eular response. However, circulating TNF bioactivity at 6 month was associated with clinical response with low TNF bioactivity in responders. A ROC analysis suggested a cut-off at 0.320 with a sensitivity at $92 \%$ and a specificity at $41 \%$.

Conclusions: After a failure of a first TNF blocker, circulating TNF bioactivity is not able to predict response to a second TNF blocker despite a lower level in responders compared to non-responders.

References:

[1] Gottenberg J-E, Brocq O, Perdriger A, Lassoued S, Berthelot J-M, Wendling D, et al. Non-TNF-Targeted Biologic vs a Second Anti-TNF Drug to Treat Rheumatoid Arthritis in Patients With Insufficient Response to a First Anti-TNF Drug: A Randomized Clinical Trial. JAMA. 2016 Sep 20;316(11):1172-80.

[2] Marotte H, Maslinski W, Miossec P. Circulating tumour necrosis factor-alpha bioactivity in rheumatoid arthritis patients treated with infliximab: link to clinical response. Arthritis Res Ther. 2005:7(1):R149-155.

Disclosure of Interest: None declared

DOI: 10.1136/annrheumdis-2017-eular.4227

\section{THU0052 LEVELS OF INFLAMMATORY SEROLOGIC BIOMARKERS IN HLA-B27 AND HLA-B15 POSITIVE PATIENTS WITH SPONDYLOARTHRITIS}

J. Londono $^{1}$, A.M. Santos ${ }^{2}$, J.C. Rueda ${ }^{2}$, P. Peña ${ }^{2}$, I. Briceño ${ }^{3}$

E.-L. Saldarriaga ${ }^{2}$, J.-I. Angarita ${ }^{2}$, E. Calvo ${ }^{4}$, M. Avila $^{2}$, N. Martinez-Rodriguez ${ }^{5}$ H. Cubides ${ }^{2}$, V. Parra ${ }^{2}$, J.F. Medina $6 .{ }^{1}$ Reumatología, Universidad de la Sabana-Hospital Militar Central, Bogotá; ${ }^{2}$ Reumatología; ${ }^{3} \mathrm{Faculltad}$ de Medicina, Grupo Genética Humana, Universidad de la Sabana, Chia; ${ }^{4}$ Departamento de Imágenes diagnósticas, Universidad Nacional, Bogotá, Colombia;

${ }^{5}$ Departamento de Investigación de Salúd Comunitaria, Hospital Infantil de México Federico Gomez, Ciudad de Mexico, Mexico; ${ }^{6}$ Unidad de Entrenamiento Clínico, Facultad de Medicina, Universidad de Navarra, Pamplona, Spain

Background: A main challenge in spondyloartritis $(\mathrm{SpA})$ management was the availability of reliable biomarkers related with disease activity, or predicting joint damage and the response to treatment. Although erythrocyte sedimentation rate (ESR) and C-reactive protein (CRP) are currently used as biomarkers for disease activity, they lack sensitivity, specificity and reproducibility. With the understanding of SpA pathogenesis, additional biomarkers like metalloproteinase 3 (MMP-3), interleukin (IL)-1a, IL-6, lipopolysaccharide-binding protein (LBP), tumour necrosis factor a (TNFa), macrophage colony stimulating factor (M-CSF), interferon gamma (INF-g), IL-17 and IL-23, had been proposed

Objectives: We aimed to evaluate the associations of MMP-3, IL-1a, IL-6, M-CSF, LPB, IL-17 and IL-23 levels in SpA patients positive for HLA-B27 or HLA-B15

Methods: 178 patients (100 men and 78 women) with SpA according to ASAS criteria were included in the study. HLA typing was performed by PCR using the Biorad ${ }^{\circledR}$ HLA-SSP ABDR plates. The levels of TNFa, IL-1a, IL-6, INF-g and IL-17 were measured by a cytometric bead-array (CBA Flex Set) using a FACS Canto II Flow CytometerÔ. Enzyme-linked immunosorbent assay (ELISA) was used to determine serum levels of IL-23, M-CSFand MMP-3. CRP and LBP levels were measured by chemiluminescence. Statistical analysis was made with SPSS v19. For comparison of quantitative variables with a normal distribution we used the Student's $t$-test. Categorical variables were presented in frequency charts and percentages, and the Chi-squared test and Fisher's exact test were used when necessary, for comparing groups. Two-tailed $\mathrm{P}$-value $<0.05$ was considered statistically significant

Results: Of the 178 patients, 70 were positive for HLA-B27, 34 for HLA-B15 and 74 had other HLA-B. According to ASAS classification criteria, 152 patients had axial SpA (axSpA) manifestations, 161 had peripheral SpA (pSpA) manifestations, and 148 patients had mixed axial and peripheral manifestations. Figure 1 shows the mean levels of inflammatory serologic biomarkers in these subgroups of patients

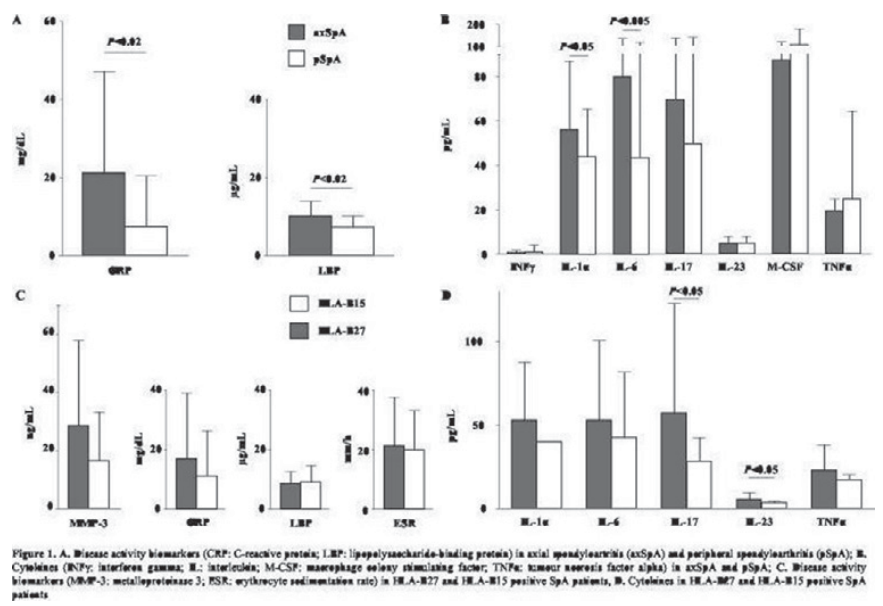

Conclusions: High levels of IL-17 and IL-23 were associated with the presence of HLA-B27, which mainly correlates with an axial presentation of the disease as 